Supporting Information

for

\title{
New unsymmetrical bisacridine derivatives non-covalently attached to quaternary quantum dots improve cancer therapy via enhancing cytotoxicity towards cancer cells and protecting normal cells
}

Joanna Pilch, ${ }^{\text {a }}$ Edyta Matysiak-Brynda, ${ }^{\mathrm{b}}$ Agata Kowalczyk, ${ }^{\mathrm{b}}$ Piotr Bujak, ${ }^{\mathrm{c}}$ Zofia Mazerska, ${ }^{\mathrm{a}}$ Anna M. Nowicka, ${ }^{\text {,* }}$ Ewa Augustin ${ }^{\mathrm{a}^{*}}$

a Faculty of Chemistry, Gdańsk University of Technology, Narutowicza Str. 11/12, 80-233 Gdańsk, Poland

${ }^{b}$ Faculty of Chemistry, University of Warsaw, Pasteura Str. 1, PL-02-093 Warsaw, Poland

c Faculty of Chemistry, Warsaw University of Technology, Noakowskiego Str. 3, 00-664 Warsaw, Poland.

*Corresponding authors: anowicka@chem.uw.edu.pl (A. Nowicka), ewa.augustin@pg.edu.pl (E. Augustin) 


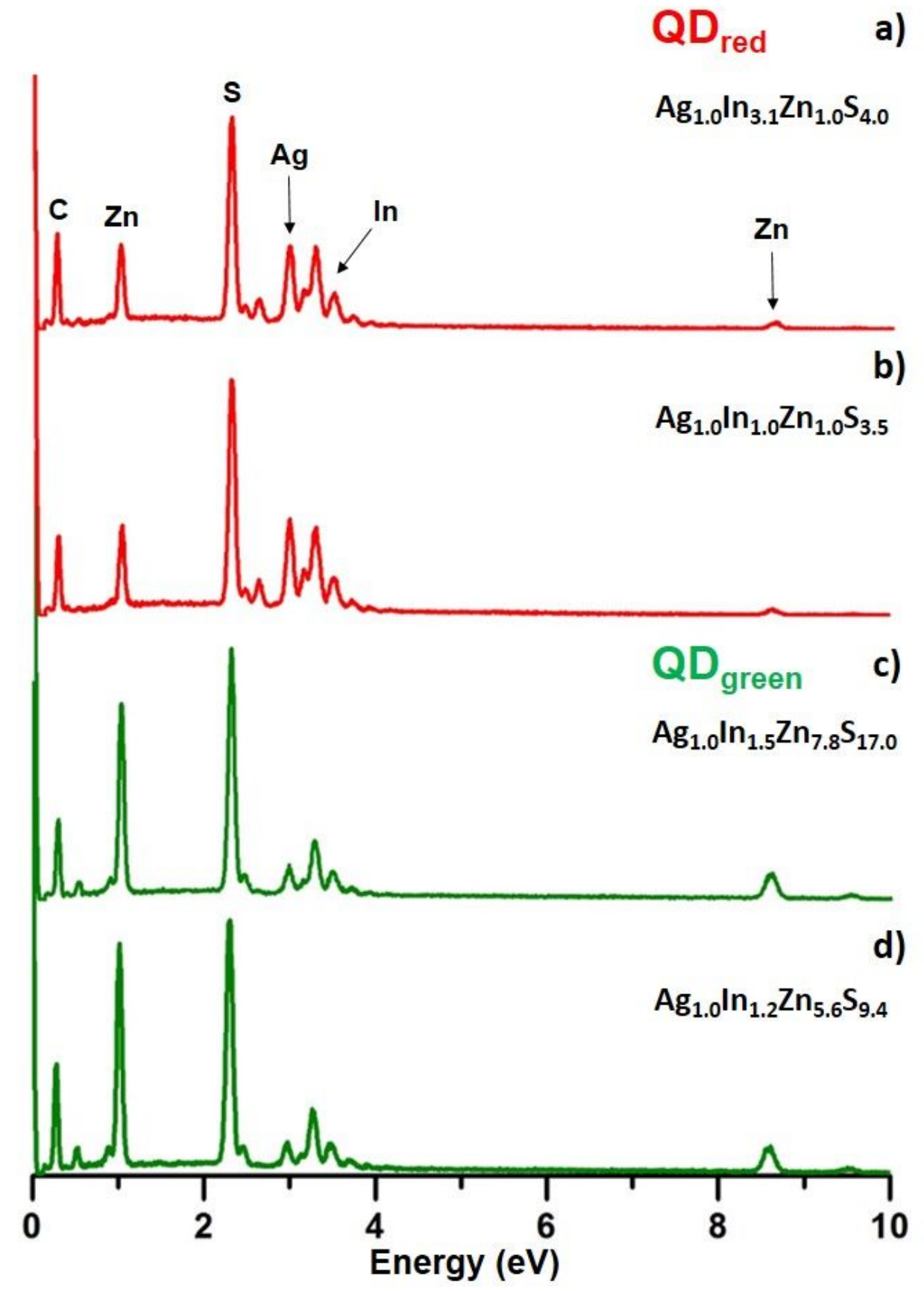

Figure S1. EDS spectra of $\mathrm{QD}_{\text {red }}$ and $\mathrm{QD}_{\text {green }}$. 

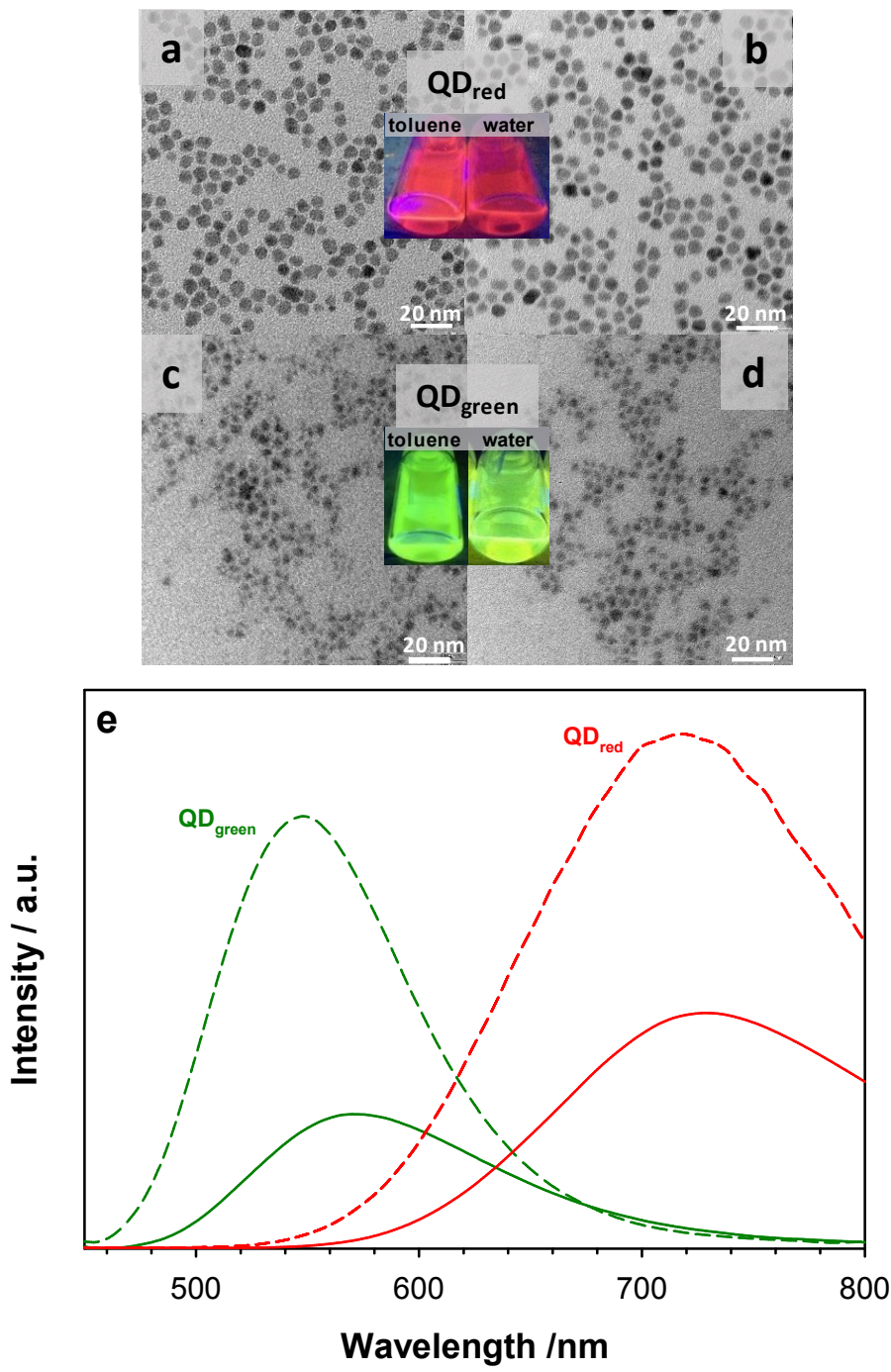

Figure S2. TEM images of Ag-In-Zn-S nanocrystals $\left(\mathrm{QD}_{\text {red }}\right.$ and $\left.\mathrm{QD}_{\text {green }}\right)$ before $(\mathrm{a}, \mathrm{c})$ and after (b, d) ligands exchange for MUA. (e) Comparison of Ag-In-Zn-S (QD red and $\left.\mathrm{QD}_{\text {green }}\right)$ nanocrystals photoluminescence spectra for dispersions in toluene (dashed lines) and water (solid lines). 


\begin{tabular}{|c|c|c|c|c|}
\hline & $\mathbf{Q D}_{\text {green }}$ & $\mathbf{Q D} \mathbf{D}_{\text {red }}$ & C-2028 & C-2045 \\
\hline $\mathrm{pH}=8.4$ & 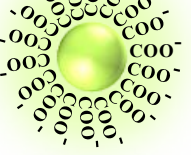 & & & \\
\hline $\mathrm{pH}=7.4$ & 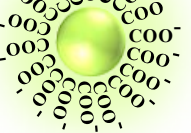 & & & \\
\hline $\mathrm{pH}=5.5$ & 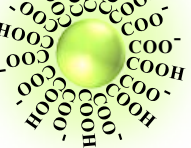 & & & \\
\hline $\mathrm{pH}=4.0$ & 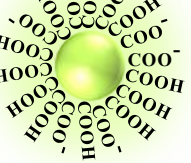 & & & \\
\hline
\end{tabular}

Figure S3. Forms of QD nanocrystals and UAs compounds at different values of $\mathrm{pH}$. 
Table S1. Assignments of bands in FTIR spectra based on ref. ${ }^{1,2}$

\begin{tabular}{|c|c|c|}
\hline Origin & Wavenumber $\left(\mathrm{cm}^{-1}\right)$ & Assignment \\
\hline \multicolumn{3}{|c|}{$Q D s$} \\
\hline $\mathrm{O}-\mathrm{H}$ & 1410 & carboxylate \\
\hline $\mathrm{C}=\mathrm{O}$ & $1555-1560$ & (carboxylic acid salt) \\
\hline \multicolumn{3}{|c|}{ UAs compounds } \\
\hline $\mathrm{C}-\mathrm{H}$ & $871-957$ & aromatic out-of-plane \\
\hline $\mathrm{C}-\mathrm{H}$ & $1050-1260$ & aromatic in-plane \\
\hline $\mathrm{C}-\mathrm{N}$ & $1090-1270$ & secondary and tertiary amine \\
\hline $\mathrm{O}-\mathrm{H}$ & 1292 & phenol \\
\hline $\mathrm{N}=\mathrm{O}$ & 1330 & nitro group \\
\hline $\mathrm{C}-\mathrm{N}$ & $1381-1384$ & aliphatic chain \\
\hline $\mathrm{C}-\mathrm{H}$ & 1447 & from $-\mathrm{CH}_{2}-$ in aliphatic chain \\
\hline $\mathrm{N}=\mathrm{O}$ & 1493 & nitro group \\
\hline $\mathrm{C}=\mathrm{N}$ & & \\
\hline $\mathrm{C}=\mathrm{C}$ & $1522-1534$ & in aromatic ring \\
\hline $\mathrm{N}-\mathrm{H}$ & $1587-1601$ & secondary amine \\
\hline $\mathrm{C}=\mathrm{C}$ & & in aromatic ring \\
\hline $\mathrm{C}=\mathrm{O}$ & $1627-1668$ & carbonyl group \\
\hline
\end{tabular}


Table S2. Hydrodynamic diameter values determined from DLS measurements and zeta potential values for UAs compounds, QD nanocrystals and QD-UAs nanoconjugates obtained in $0.02 \mathrm{M}$ PBS buffer at various $\mathrm{pH}$.

\section{a: Hydrodynamic diameter (nm)}

\begin{tabular}{|c|c|c|c|c|}
\hline C-2028* & \multicolumn{4}{|c|}{$2.1 \pm 0.4$} \\
\hline C-2045* & \multicolumn{4}{|c|}{$2.2 \pm 0.3$} \\
\hline $\mathbf{Q D}_{\text {red }}$ & \multicolumn{4}{|c|}{$9.1 \pm 0.8$} \\
\hline $\mathbf{Q D} \mathbf{D}_{\text {green }}$ & \multicolumn{4}{|c|}{$9.4 \pm 0.7$} \\
\hline & $p H=4.0$ & $p H=5.5$ & $p H=7.4$ & $p H=8.4$ \\
\hline $\mathrm{QD}_{\text {red }}-\mathrm{C}-2028$ & $9.2 \pm 0.5$ & $10.2 \pm 0.9$ & $10.8 \pm 0.8$ & $10.9 \pm 0.9$ \\
\hline$Q_{\text {red }}-C-2045$ & $9.4 \pm 0.3$ & $11.9 \pm 0.5$ & $12.6 \pm 0.9$ & $12.4 \pm 0.6$ \\
\hline $\mathrm{QD}_{\text {green }}-\mathrm{C}-2028$ & $9.9 \pm 0.6$ & $10.1 \pm 0.8$ & $11.9 \pm 0.7$ & $11.8 \pm 0.8$ \\
\hline $\mathrm{QD}_{\text {green }}-\mathrm{C}-2045$ & $11.4 \pm 0.7$ & $11.7 \pm 0.9$ & $12.5 \pm 0.4$ & $12.7 \pm 0.6$ \\
\hline \multicolumn{5}{|c|}{ b: Zeta potential values } \\
\hline & $p H=4.0$ & $p H=5.5$ & $p H=7.4$ & $p H=8.4$ \\
\hline C-2028 & $-15.3 \pm 3.4$ & $-14.3 \pm 2.5$ & $-19.3 \pm 1.2$ & $-18.1 \pm 1.5$ \\
\hline $\mathrm{C}-2045$ & $-20.4 \pm 1.6$ & $-17.7 \pm 1.7$ & $-13.6 \pm 2.2$ & $-20.5 \pm 1.1$ \\
\hline $\mathbf{Q D}_{\text {red }}$ & $-13.6 \pm 1.5$ & $-34.2 \pm 2.9$ & $-34.1 \pm 2.5$ & $-34.8 \pm 2.8$ \\
\hline $\mathbf{Q D}_{\text {green }}$ & $-19.2 \pm 1.8$ & $-28.9 \pm 1.8$ & $-38.5 \pm 1.9$ & $-37.5 \pm 2.5$ \\
\hline QD $_{\text {red }}-\mathrm{C}-2028$ & $-5.5 \pm 1.1$ & $-31.1 \pm 1.9$ & $-33.4 \pm 2.6$ & $-30.2 \pm 2.5$ \\
\hline QD $_{\text {red }^{-}}-\mathbf{C - 2 0 4 5}$ & $-7.5 \pm 1.3$ & $-30.1 \pm 1.5$ & $-43.2 \pm 2.5$ & $-36.7 \pm 1.5$ \\
\hline QD ${ }_{\text {green }}-C-2028$ & $-18.5 \pm 2.5$ & $-30.3 \pm 1.8$ & $-39.0 \pm 1.5$ & $-37.5 \pm 2.7$ \\
\hline QD ${ }_{\text {green }}-C-2045$ & $-24.0 \pm 2.0$ & $-26.8 \pm 1.3$ & $-40.1 \pm 2.5$ & $-36.6 \pm 2.2$ \\
\hline
\end{tabular}

*the diameters of UAs compounds were determined with the help of the ChemSketch program where it was assumed that the molecules are circular 
Below we present the representative picture and the tables with the statistic for the in vivo test. Table S3. presents the data for Figure 9. There are the mean tumor volume and the standard deviations of these values.

Table S3.

\begin{tabular}{|c|c|c|c|c|c|c|c|c|c|c|c|c|c|c|}
\hline \multirow{2}{*}{ Day } & \multicolumn{2}{|c|}{ Glucose } & \multicolumn{2}{|c|}{ Irinotecan } & \multicolumn{2}{|c|}{ C-2028 } & \multicolumn{2}{|c|}{ C-2045 } & \multicolumn{2}{|c|}{$\mathbf{Q D}_{\text {red }}$} & \multicolumn{2}{|c|}{$\mathrm{QD}_{\text {red }}-\mathrm{C}-2028$} & \multicolumn{2}{|c|}{$\mathrm{QD}_{\text {red }}-\mathrm{C}-2045$} \\
\hline & mean & $S D$ & mean & $S D$ & mean & $S D$ & mean & $S D$ & mean & $S D$ & mean & $S D$ & mean & $S D$ \\
\hline 1 & 117.5 & 12.6 & 116.7 & 14.1 & 114.4 & 13.6 & 116.4 & 22.3 & 117.7 & 14.8 & 117.5 & 17.0 & 115.0 & 20.4 \\
\hline 4 & 218.2 & 29.0 & 163.8 & 27.1 & 173.0 & 15.6 & 200.3 & 27.0 & 206.7 & 32.0 & 215.0 & 37.8 & 236.1 & 50.1 \\
\hline 6 & 291.1 & 41.3 & 221.3 & 38.5 & 265.7 & 30.6 & 309.6 & 35.0 & 324.2 & 48.8 & 371.2 & 57.3 & 331.2 & 61.1 \\
\hline 8 & 448.8 & 57.0 & 280.1 & 48.7 & 389.1 & 38.9 & 427.7 & 42.0 & 466.5 & 52.6 & 572.4 & 85.2 & 529.3 & 99.0 \\
\hline 10 & 756.5 & 111.1 & 352.1 & 54.6 & 542.0 & 54.9 & 615.7 & 50.1 & 718.7 & 93.4 & 829.3 & 111.8 & 861.1 & 169.4 \\
\hline 12 & 1010.5 & 112.4 & 434.5 & 62.7 & 667.7 & 81.8 & 739.0 & 52.3 & 881.0 & 115.5 & 862.1 & 117.6 & 1015.2 & 176.1 \\
\hline 14 & 1341.9 & 138.0 & 508.5 & 71.4 & 810.9 & 100.3 & 984.4 & 92.3 & 1156.2 & 163.2 & 1136.1 & 153.5 & 1306.0 & 213.7 \\
\hline 17 & 1523.6 & 163.4 & 431.1 & 60.9 & 887.4 & 136.9 & 1088.5 & 114.2 & 1424.8 & 248.9 & 1181.6 & 139.5 & 1577.9 & 234.6 \\
\hline
\end{tabular}

The Figure below presents the same results in the form of a bar graph.

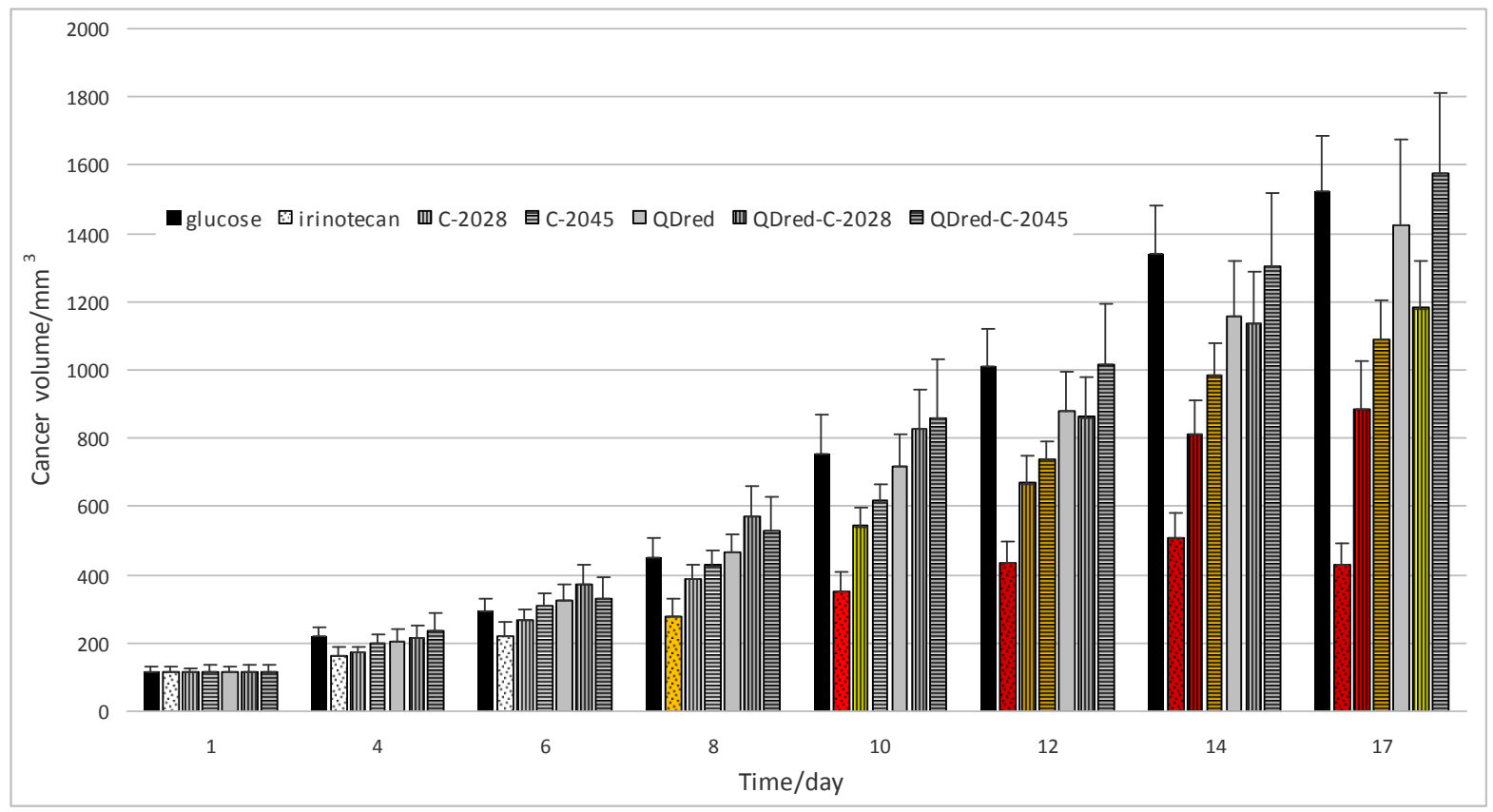

Figure S4. In vivo antitumor efficacy of C-2028 and C-2045 non-covalently attached to $\mathrm{QD}_{\text {red, }}$ $\mathrm{QD}_{\text {red }}$ and pure bisacridines in HCT116 xenografts in nude mice. Positive control: irinotecan.

Furthermore, we present in Table S4 the values of Student's t-statistics for the significance of tumor volume differences in relation to control for the selected days of experiments. Levels of significance are presented additionally in appropriate colors and with sign "+" or " **".

The same code of colors is used in Figure S4. 
Table S4.

\begin{tabular}{|c|c|c|c|c|c|c|c|c|c|c|c|c|c|c|}
\hline \multirow{3}{*}{$\frac{\text { Day }}{1}$} & \multicolumn{2}{|c|}{ Irinotecan } & \multicolumn{2}{|c|}{ C-2028 } & \multicolumn{2}{|c|}{ C-2045 } & $\mathbf{Q D}_{\text {red }}$ & \multicolumn{2}{|c|}{$\mathrm{QD}_{\text {red }}-\mathrm{C}-2028$} & \multicolumn{2}{|c|}{$\mathrm{QD}_{\text {red }}-\mathrm{C}-2045$} & \multirow{3}{*}{$\mathrm{p}$ value } & \multirow{3}{*}{$\begin{array}{c}\text { critical } \\
\text { value }\end{array}$} & \multirow{3}{*}{ code } \\
\hline & t stat. & signif. & t stat. & signif. & t stat. & signif. & t stat. signif. & t stat. & signif. & t stat. & signif. & & & \\
\hline & 0.040 & & 0.158 & & 0.044 & & 0.010 & 0.000 & & 0.106 & & & & \\
\hline 4 & 1.258 & & 1.166 & & 0.415 & & 0.252 & 0.066 & & 0.317 & & $<0.1$ & 1.316 & + \\
\hline 6 & 1.135 & & 0.437 & & 0.309 & & 0.496 & 1.120 & & 0.543 & & $<0.05$ & 1.708 & $*$ \\
\hline 8 & 2.037 & $*$ & 0.757 & & 0.264 & & 0.209 & 1.207 & & 0.724 & & $<0.01$ & 2.485 & $* *$ \\
\hline 10 & 2.751 & $* *$ & 1.458 & + & 0.966 & & 0.235 & 0.430 & & 0.518 & & $<0.001$ & 3.450 & $* * *$ \\
\hline 12 & 3.819 & $* * *$ & 2.177 & $*$ & 1.836 & $*$ & 0.751 & 0.855 & & 0.023 & & & & \\
\hline 14 & 4.539 & $* * *$ & 2.746 & $* *$ & 1.878 & $*$ & 0.833 & 0.945 & & 0.142 & & & & \\
\hline 17 & 5.167 & $* * *$ & 2.692 & $* *$ & 1.915 & $*$ & 0.333 & 1.440 & + & 0.189 & & & & \\
\hline
\end{tabular}

(1) Coates, J. Interpretation of Infrared Spectra, a Practical Approach, in: Meyers, R.,A.; (Ed.), Encyclopedia of Analytical Chemistry, John Wiley\&Sons Ltd, Chichester, 2000, pp.10815-10837.

(2) Thomas, G. J.; Kyogoku, Y. Biological Science, in: Brame, E. G.; Grasselli, J.G.; (Eds.), Infrared and Raman Spectroscopy (part C), Marcel Dekker, New York, 1977, pp. 717-861. 